Audiology

Neurotology
Audiol Neurotol 2019;24:309-321

DOI: $10.1159 / 000504593$
Received: June 4, 2019

Accepted after revision: November 5, 2019

Published online: January 6, 2020

\title{
Amplitude Modulated Noise for Tinnitus Suppression in Tonal and Noise-Like Tinnitus
}

\author{
Stefan Schoisswohl ${ }^{\mathrm{a}}$ Johannes Arnds ${ }^{\mathrm{a}}$ Martin Schecklmann ${ }^{\mathrm{a}}$ \\ Berthold Langguth $^{a}$ Winfried Schlee $^{a}$ Patrick Neff ${ }^{a, b}$ \\ a Department of Psychiatry and Psychotherapy, University of Regensburg, Regensburg, Germany; \\ bUniversity Research Priority Program "Dynamics of Healthy Aging," University of Zurich, Zurich, Switzerland
}

\section{Keywords}

Tinnitus · Noise · Residual inhibition · Amplitude

modulation $\cdot$ Bandpass filter

\begin{abstract}
Background: The phenomenon of short-term tinnitus suppression by different forms of acoustic stimulation is referred to as residual inhibition ( $\mathrm{RI})$. RI can be triggered in the majority of tinnitus cases and was found to be depending on the used intensity, length or types of sounds. Past research already stressed the impact of noise stimulation as well as the superiority of amplitude modulated (AM) pure tones at the individual tinnitus frequency for RI in tonal tinnitus. Recently a novel approach for the determination of noise-like tinnitus characteristics was proposed. Objectives: The aim of the present study was to investigate whether in participants with noise-like tinnitus RI can be increased by AM noise stimuli according to the individual tinnitus frequency range. Methods: For this purpose the individual tinnitus characteristics (noise-like and tonal tinnitus) of 29 people affected by tinnitus (mean age $=55.59$, 7 females, mean tinnitus duration $=159.97$ months) were assessed via customizable noiseband matching. The objective was to generate bandpass fil-
\end{abstract}

\begin{tabular}{ll}
\hline KARGER & $\begin{array}{l}\text { (c) } 2020 \text { The Author(s) } \\
\text { Published by S. Karger AG, Basel }\end{array}$ \\
E-Mail karger@karger.com & This article is licensed under the Creative Commons Attribution- \\
www.karger.com/aud & $\begin{array}{l}\text { NonCommercial-NoDerivatives 4.0 International License (CC BY- } \\
\text { NC-ND) (http://www.karger.com/Services/OpenAccessLicense). } \\
\text { Usage and distribution for commercial purposes as well as any dis- } \\
\text { tribution of modified material requires written permission. }\end{array}$
\end{tabular}

tered stimuli according to the individual tinnitus sound (individualized bandpass filtered [IBP] sounds). Subsequently, various stimuli differing in bandpass filtering and AM were tested with respect to their potential to induce RI. Participants were acoustically stimulated with 7 different types of stimuli for 3 min each and had to rate the loudness of their tinnitus after each stimuli. Results: Results indicate a general efficacy of noise stimuli for the temporary suppression of tinnitus, but no significant differences between AM and unmodulated IBP. Significantly better effects were observed for the subgroup with noise-like tinnitus ( $n=14)$, especially directly after stimulation offset. Conclusions: The study at hand provides further insights in potential mechanisms behind RI for different types of tinnitus. Beyond that, derived principles may qualify for new or extend current tinnitus sound therapies.

(C) 2020 The Author(s)

Published by S. Karger AG, Basel

\section{Introduction}

Chronic subjective tinnitus is defined as the permanent perception of a sound such as ringing or hissing in the absence of an external or internal source of noise. Ap- 
proximately $10-15 \%$ of the population in industrial countries experience this phantom sound [Langguth et al., 2013; Erlandsson and Dauman, 2013; Heller, 2003; Hall et al., 2011]. Causes for the development of tinnitus are divergent and not completely understood, though most commonly tinnitus occurs towards cochlear damages due to noise trauma [Langguth et al., 2013]. In the majority of cases, the perceived tinnitus pitch is in accordance with the frequency spectrum of hearing loss (HL) [Basile et al., 2013; Roberts et al., 2008]. As a consequence of decreased or absent auditory input and the subsequent deficiency of neural input, maladaptive pathological changes in the auditory pathway are formed, which lead to the perception of a "phantom sound" defined as tinnitus [Eggermont, 2007; Eggermont and Tass, 2015; Eggermont and Roberts, 2012]. Neurophysiological investigations of tinnitus were able to demonstrate hyperactivity in auditory brain areas [Farhadi et al., 2010; Folmer, 2007] as well as aberrant oscillatory brain activity and connectivity patterns [Schlee et al., 2009, 2014; Moazami-Goudarzi et al., 2010; Mohan et al., 2016]. Available treatment options have only limited efficacy and to date there is no cure available [Baguley et al., 2013]. Auditory stimulation is one potential treatment approach for tinnitus, but also provides insights to basic mechanisms of tinnitus [Roberts et al., 2008; Fournier et al., 2018].

Almost half a century ago, Feldmann [1971, 1983] investigated the phenomenon of short-term tinnitus suppression after sound stimulation. This temporary suppression is referred to as "residual inhibition" (RI), which manifests in individual suppression patterns (i.e., duration, depth and shape) and can be triggered in $60-80 \%$ of tinnitus cases [Roberts, 2007; Vernon and Meikle, 2003]. Various recent studies scrutinized RI in more depth. Data from several investigations suggest the effects of RI to be more prominent with sounds close or within the individual tinnitus frequency spectrum [Roberts et al., 2006, 2008; Schaette et al., 2010]. Factors including duration or intensity of the stimuli also affect RI [Terry et al., 1983; Norena et al., 2002; Vernon and Fenwick, 1984; Neff et al., 2017]. In contrast, the underlying neurophysiological mechanisms of RI are not clearly understood yet [Roberts, 2007; Galazyuk et al., 2019]. Most recent work suggests that tinnitus suppression through sound stimulation is related to reduced spontaneous firing of central auditory neurons [Galazyuk et al., 2017, 2019].

The importance of stimulation intensity and frequency was verified in a recent work from Fournier et al. [2018], who developed a novel approach for RI testing described as Minimum RI Level. Thereby, people had to adjust the intensity of customized stimuli up to the point where their tinnitus is suppressed during a given interval after the offset of the stimulus. Results show an occurrence of RI in $86.7 \%$ of people with tinnitus by using this method [Fournier et al., 2018].

Despite noise-like tinnitus perception in many cases, to the best of our knowledge, none of the previous mentioned studies included a genuine matching for noise-like tinnitus, that is, determination of noise band-width [Roberts et al., 2006; Fournier et al., 2018].

Recently Henry et al. [2013] proposed a novel approach for tinnitus matching procedures taking into consideration the tinnitus type. In addition to the determination of the centre frequency, people with tinnitus were also able to adjust the band-width of their tinnitus [Henry et al., 2013]. Here we aim to use both frequency and band-with information to develop individualized stimuli, especially for people with noise-like tinnitus, for the investigation of RI.

Previous studies investigating the effects of differently modulated sounds on RI revealed that amplitude modulated (AM) tones near or at the individual tinnitus frequency result in larger RI [Reavis et al., 2012; Tyler et al., 2014]. Furthermore, differential results for specific amplitude modulation rates were observed [Neff et al., 2017, 2019].

The experiment aims at investigating the effects of different noise stimuli with and without AM on RI. The overarching goal is to establish new acoustic stimulation techniques for basic RI research as well as generating principles for possible future sound stimulation concepts with the AM stimulus class. For this purpose, the individual tinnitus characteristics are assessed via noise-band matching as suggested by Henry et al. [2013] in order to create personalized stimuli for RI examination.

Previous studies in the field of RI already emphasized the impact of noise stimulation on tinnitus perception in tonal tinnitus [Henry et al., 2013; Fournier et al., 2018; Roberts et al., 2006, 2008]. To the best of our knowledge, none of the existing experiments systematically investigated these noise stimulation methods, in particular the application of AM or bandpass filters (BP) to noise stimuli, in noise-like tinnitus.

According to this, the current experiment represents the first attempt to investigate the effects of an administration of individualized BP settings (IBP) and different rates of AM (10 and $40 \mathrm{~Hz})$ to white noise (WN) on RI.

These stimulation methods are furthermore merged to a novel combinatory approach to apply IBP and AM to $\mathrm{WN}$ simultaneously and scrutinize its efficacy in RI. 
Additionally, each of the used stimuli was examined with regards to induced arousal and valence as rated by the participants, since differences in stimuli evaluation could potentially affect tinnitus suppression.

Besides the assumption of the efficacy of all deployed noise stimuli in short-term tinnitus inhibition (in both noise-like and tonal tinnitus), we expect that IBP differs in its effects on RI from unadjusted WN. We hypothesized that the IBP would result in different strengths of residual tinnitus suppression compared to WN. Yet, given the lack of previous studies we are not able to define a directed hypothesis here. Furthermore, building on the insights of previous work, we hypothesize that stimulations with AM noise (filtered or unchanged) result in larger RI than their unmodulated counterparts.

\section{Methods}

\section{Participants}

The sample for this experiment consisted of 29 participants (7 female) between 18 and 75 years with noise-like $(n=14)$ or tonal tinnitus $(n=15)$ with a tinnitus duration of $>6$ months. Participants were recruited from the Interdisciplinary Tinnitus Centre in Regensburg, Germany. For detailed sample characteristics see Table 1. Primary inclusion criteria were no somatic, mental health or neurological conditions and no current intake of psychotropic medications or substances. Alike, participants were not allowed to participate in other tinnitus-related studies. The methods and the procedures used in this study were examined and approved by the local Ethics Committee of the University of Regensburg (16-1010061). All participants were sufficiently informed about the aim, methods, and duration of the study, possible side effects, and gave written informed consent prior to the start of the experiment.

\section{Psychometry}

Each participant filled in an online survey composed of German versions of the Tinnitus Handicap Inventory [Newman et al., 1994; Kleinjung et al., 2007], the Tinnitus Questionnaire (TQ) [Goebel and Hiller, 1994; Hallam et al., 1988], a brief version of the Hyperacusis Questionnaire (mini-HQ9) [Goebel et al., 2013] and the Tinnitus Sample Case History Questionnaire for tinnitus-related clinical and demographic information [Langguth et al., 2007].

\section{Audiometry}

For the purpose of individual hearing threshold determination, frequencies ranging from $125 \mathrm{~Hz}$ to $8 \mathrm{kHz}$ in octave steps including semi-octave steps between 0.5 and 1 (i.e., $0.75 \mathrm{kHz}$ ), 1 and 2 (i.e., $1.5 \mathrm{kHz}$ ), 2 and 4 (i.e., $3 \mathrm{kHz}$ ) and 4 and $8 \mathrm{kHz}$ (i.e., $6 \mathrm{kHz}$ ) were quantified with a clinical audiometer (Madsen Midimate 622D; GN Otometrics, Denmark). Sennheiser HDA 2000 headphones (Sennheiser, Germany) were used for audiometric measurements, subsequent tinnitus matching and acoustic stimulation. Minimum masking level (MML) was assessed by increasing the loudness of a WN sound (Madsen Midimate 622D; GN Otometrics, Denmark) until their tinnitus was completely masked.

\section{Tinnitus Matching}

In order to ascertain participants individual tinnitus pitch, the Method of Adjustment approach [Henry et al., 2013] was performed with a custom-made MAX application (MAX 7; Cycling'74, USA) together with a modular hardware controller ( $\mathrm{Pa}$ lette Expert Kit; Palette, Canada). The matching procedure's steps were in accordance with the order within the Tinnitus Tester procedure [Roberts et al., 2008] with an additional test for octave confusion at the end. Prior to tinnitus matching, participants were asked to vocalize or describe their tinnitus to distinguish between noise-like and tonal tinnitus types as indicated in the recruiting process. Following on that, they were instructed and trained for the process of tinnitus matching. Parameters examined by the matching procedure were as follows: tinnitus frequency, respectively centre frequency for noise-like tinnitus $(\mathrm{Hz})$, tinnitus loudness $(\mathrm{dB})$ and tinnitus laterality $(0=$ left ear; $127=$ right ear; thus a value of 63 describes a bilateral tinnitus). Control units of the matching controller were labelled accordingly. The step size of frequency dial was marginally below a semitone and ranged from $40 \mathrm{~Hz}$ to $16 \mathrm{kHz}$. For tonal tinnitus matching, a $3 \mathrm{kHz}$ pure tone with comfortable loudness was set as a starting point, followed by an adjustment of the frequency by the participants to determine their individual tinnitus frequency. Finally, tinnitus loudness and laterality were adjusted with the matching controller to complete the matching procedure. In case of noise-like tinnitus, the starting sound was a filtered broadband noise (bandwidth: 1/3 octave of centre frequency). Participants were able to adjust the centre frequency of the noise and also the bandwidth of the filter settings according to their individual tinnitus noise. Subsequently, loudness and laterality were identified just as with the pure tone matching. Finally, participants rated the agreement of their tinnitus with the matched sound on a 1-10 scale. To assess individuals sensation level (SL), the hearing threshold of the frequency next to the individual tinnitus frequency or centre frequency was used (i.e., stepping down to the next lower frequency. For example, if the individual tinnitus frequency was $7.4 \mathrm{kHz}$, the hearing threshold at $7 \mathrm{kHz}$ was investigated). The matching procedure was repeated after the acoustic stimulation block of the experiment.

\section{Acoustic Stimulation}

Seven different modified noise stimuli were created in MATLAB (Matlab R2015a; Mathworks, Natick, MA, USA) and utilised for a 3 min acoustic stimulation with an intensity of $60 \mathrm{~dB}$ SL. Stimuli set consisted of unmodified WN, WN with AM rates at $10 \mathrm{~Hz}(\mathrm{WN10})$ and $40 \mathrm{~Hz}$ (WN40), as well as a IBP with the same modulation rates (IBP, IBP10, IBP40). BP width was set according to the matching results in noise-like tinnitus participants. In participants with tonal tinnitus, the previously matched individual tinnitus pitch was used to deploy an IBP to WN with a range of one octave [Pantev et al., 2012]. Furthermore an IBP WN with $10 \mathrm{~Hz}$ AM rates at MML intensity (IBP10_MML) was used for acoustic stimulation in order to contrast SL and MML. Acoustic stimulation was conducted in a randomized order for each session with a maximum loudness of $80 \mathrm{dBSPL}$ diotically over the headphones. If participants experienced discomfort, they were able to stop the stimulation and experimental procedures at any time. Following a 3-minute stimulation for each stimulus, participants evaluated their tinnitus loudness (\%) in comparison to prior the particular stimulation on a numeric rating scale ( $0 \%$ up to $140 \%$ in $10 \%$ steps) at 7 different points in time $(0,30,60,90,120,150$ and $180 \mathrm{~s}$ after 


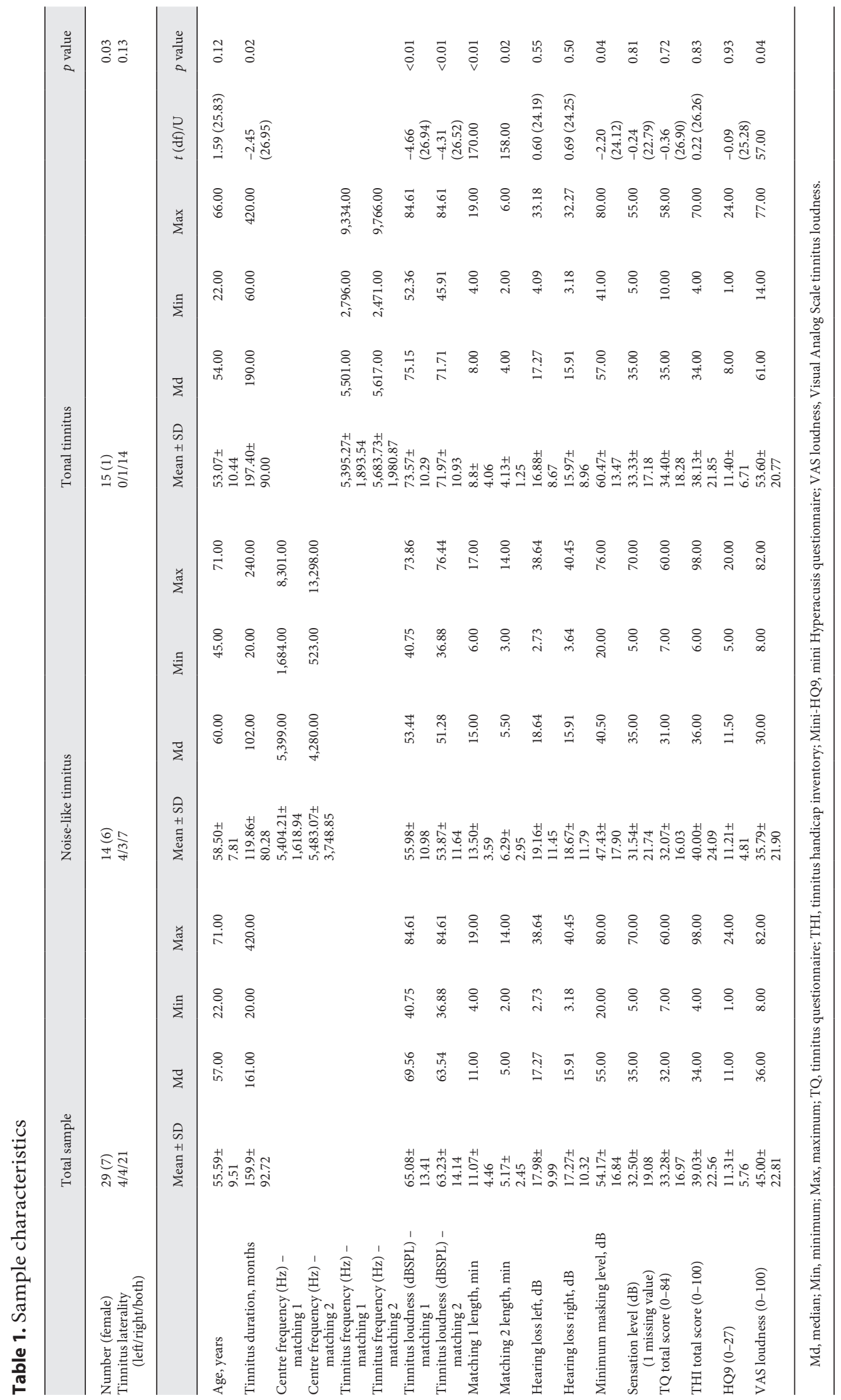




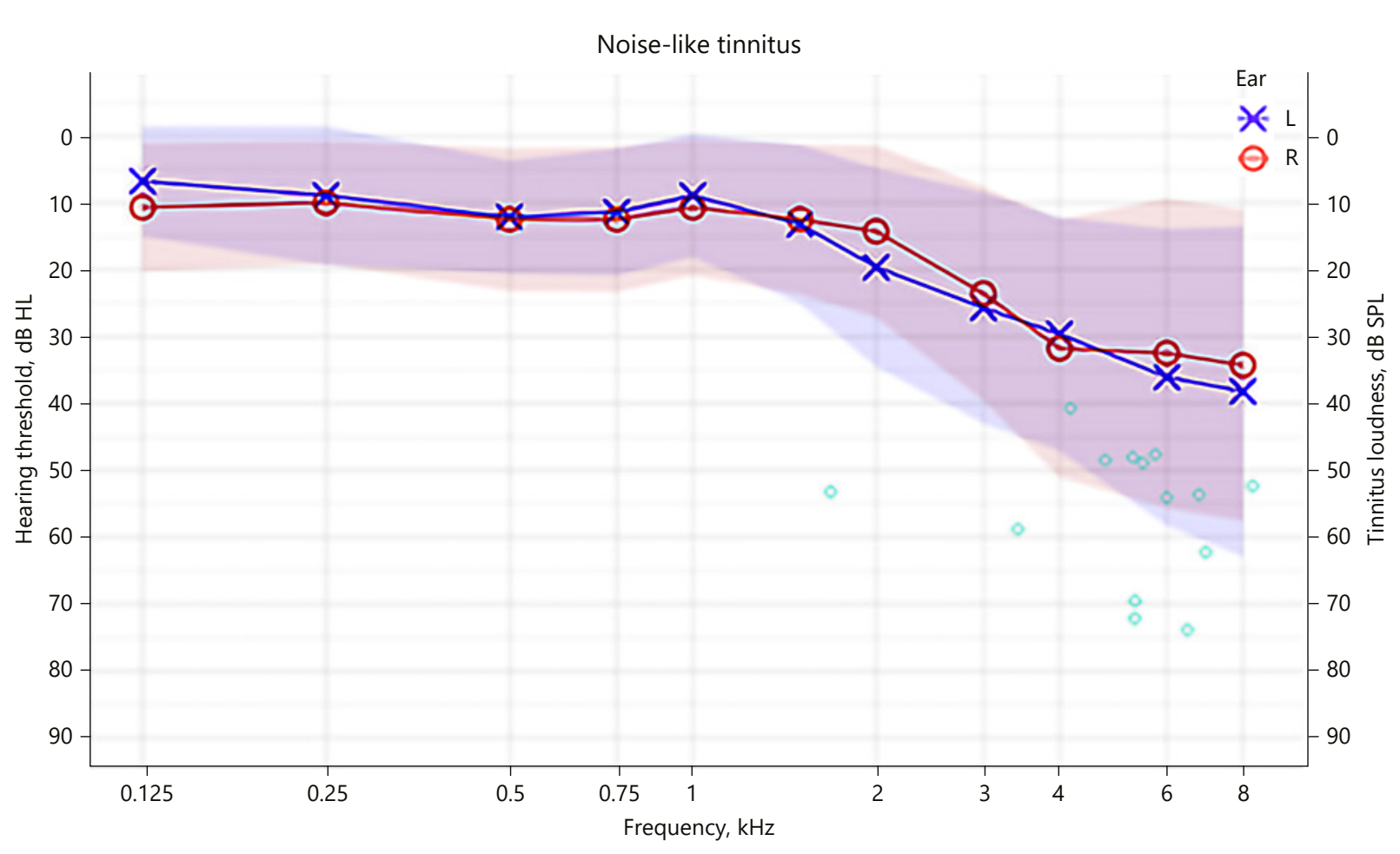

Tonal tinnitus

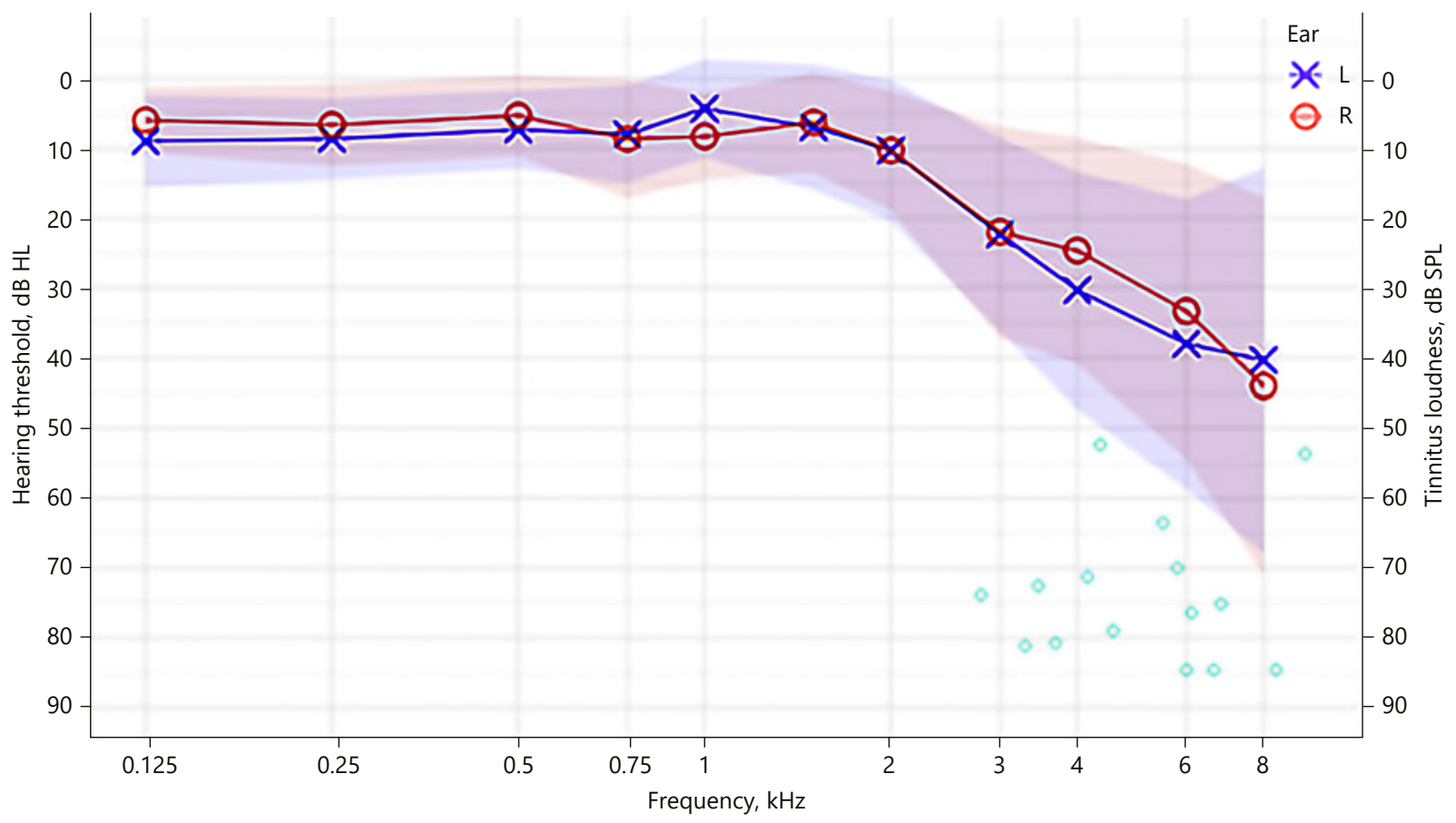

Fig. 1. Audiometry and Tinnitometry. Audiometric measurement results for both ears together with individual tinnitus frequency (i.e., centre frequency of the IBP) and loudness as identified by tinnitus matching split for noise-like and tonal tinnitus. It should be noted, that tinnitus/centre frequency overlaps with the frequencies of HL. HL, hearing loss. 


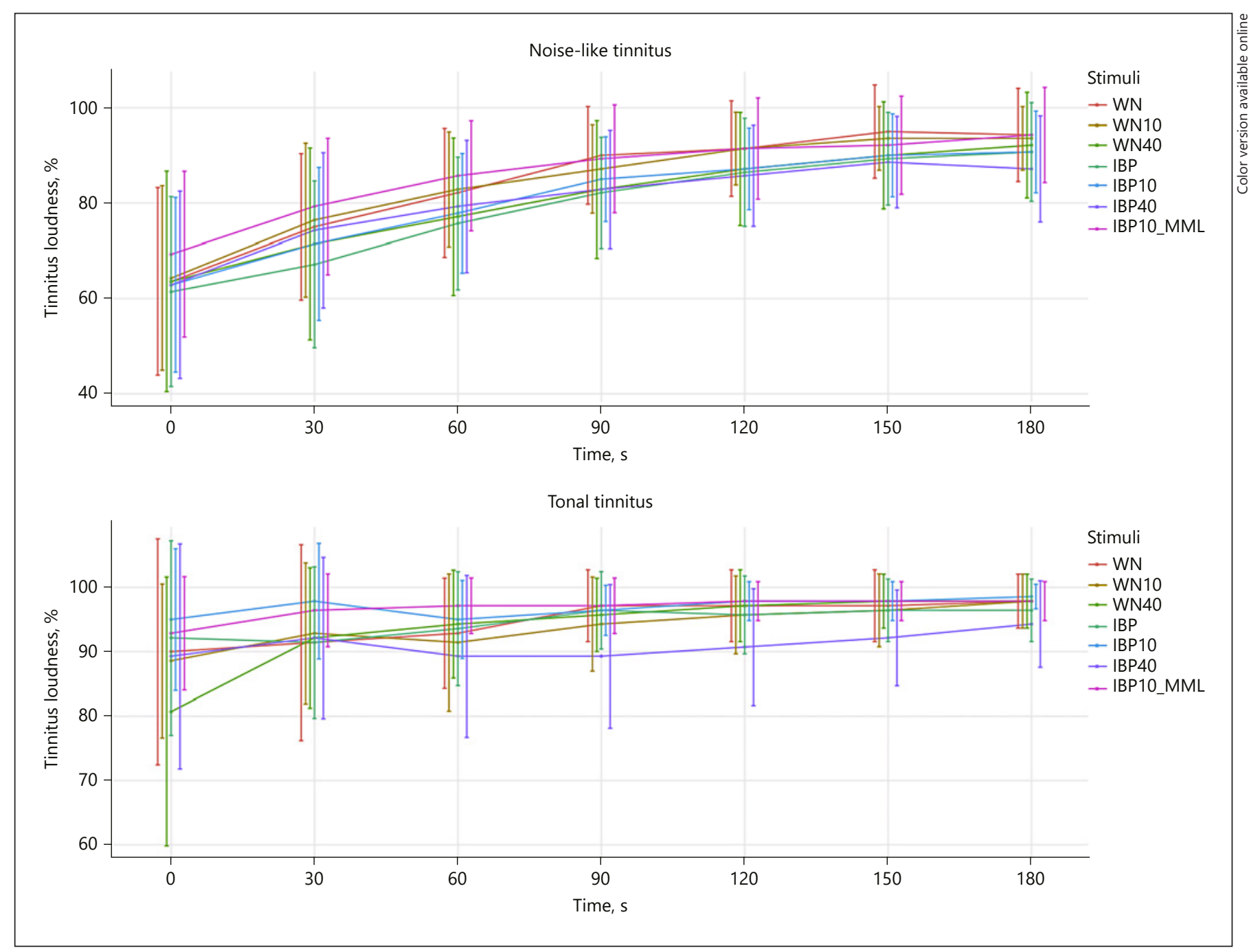

Fig. 2. Tinnitus loudness time curve split by group. For each stimulus the tinnitus loudness rating over all time points is plotted separated for noise-like and tonal tinnitus (CIs at 95\% shown as brackets). Overall, each stimulus was able to suppress tinnitus loudness (cf. online suppl. Table S1). In terms of suppression averaged over time but also at T0, stimulus IBP appeared to produce the strongest effect on loudness in the noise-like tinnitus group, whereas in the tonal group, stimulus IBP40 induced the lowest tinnitus loudness on average. However, directly after stimulation WN40 showed the strongest suppression. WN, white noise; MML, minimum masking level; IBP, individualized bandpass filtered. stimulation offset). Moreover, participants rated the induced valence and arousal of each single stimulus with pictorial manikin scales [Bradley and Lang, 1994].

\section{Statistical Analysis}

All statistical analyses were performed using the statistic software R ( $\mathrm{R}$ version 3.4.3; R Foundation for Statistical Computing, Austria) and the packages "psych," "emmeans," "sjstats," and "lme4." Tinnitus loudness and stimulus evaluation data were analysed by means of linear mixed effect models for each dependent variable denoted as response (tinnitus loudness, valence, arousal). Potential Models were compared with Likelihood Ratio Tests in a step-wise selection approach [Harrison et al., 2018]. Following predictors as well as their interactions were tested in the model fitting procedure: condition (stimuli used; see acoustic stimulation section), group (noise-like tinnitus, tonal tinnitus), time $(0,30,60$, 90, 120, 150, $180 \mathrm{~s}$ after stimulation end), gender (male, female), age, tinnitus duration, tinnitus loudness (according to first tinnitus matching), MML and tinnitus distress (TQ sum score). The proportion of explained variance was identified by marginal (variance of the fixed effects) and conditional (variance of fixed and random effects) $R^{2}$ [Nakagawa et al., 2017]. In any of the fitted models, the participant (id) was treated as a random effect. Fixed effects of the final model were tested via expected mean square approach. Posthoc Tukey tests were calculated to contrast responses for condition and group. In order to test for a potential bias due to the sequence 
of the stimuli used for acoustic stimulation (position effect), a median split was conducted on the positions variable and differences in means were then tested with Student $t$ tests.

Analysis of descriptive group differences (noise-like vs. tonal tinnitus) for parametric variables was done by the means of twosample $t$ tests. In case of violation of normal distribution and homoscedasticity, non-parametric testing via independent sample Mann-Whitney U tests was used. Categorical data was analyzed by Fisher's exact tests, due to cell frequencies below 5 in all variables.

Reliability for the matching procedure (between first and second matching round) was assessed via Pearson correlations, or rather Spearman correlations in case of a violation of normal distribution, for tinnitus loudness and tinnitus or centre frequency. Statistical significance was defined as $p \leq 0.05$ for all analysis.

\section{Results}

\section{Participant Characteristics}

Demographic and clinical characteristics for the whole study sample and for tinnitus sub-groups (noise-like and tonal tinnitus) can be found in Table 1. A Fisher's exact test was able to identify a significant association between gender and the type of tinnitus. In the group with tonal tinnitus the proportion of female participants was significantly lower $(p=0.03)$. Statistical testing revealed significant differences in terms of tinnitus duration and subjective rating of tinnitus loudness (VAS loudness), with the noise-like tinnitus group showing a shorter duration of tinnitus $\left(t_{[26.95]}=-2.45, p=0.02\right)$ and evaluating their tinnitus loudness lower $(U=57.00, p=0.04)$. Further, no differences were found in TQ $\left(t_{[26.90]}=-0.36, p=0.72\right)$, Tinnitus Handicap Inventory $\left(t_{[26.26]}=0.22, p=0.83\right)$ or HQ9 $\left(t_{[25.28]}=-0.09, p=0.93\right)$ scores among the 2 subgroups.

\section{Audiometry and Tinnitometry}

Table 1 shows audiometric and tinnitus matching results with a significant lower tinnitus loudness (corresponding with subjective loudness rating; see the descriptives section above) for both matching procedures (matching 1: $t_{[26.94]}=-4.66, p<0.01$; matching $2: t_{[26.52]}=$ $-4.31, p<0.01)$ and $\operatorname{MML}\left(t_{[24.12]}=-2.20, p=0.04\right)$ in the group of noise-like tinnitus. On the basis of a consolidation of these audiometric and tinnitometric findings, Figure 1 indicates an overlap of tinnitus frequency with the frequency of HL. As might be expected, the length of the first and second matching process was significantly shorter in the tonal tinnitus group (cf. Table 1). Mean HL difference for both ears were not significantly different between groups (left: $t_{[24.19]}=0.60, p=0.55$; right: $t_{[24.25]}=$ $0.69, p=0.50)$. In both groups, the HL was more pronounced on the left side.
Table 2. Fixed effect testing

\begin{tabular}{llrrr}
\hline & numDF & denDF & \multicolumn{1}{c}{$F$} & $p$ value \\
\hline Condition & 6.00 & $1,392.00$ & 3.35 & $<0.01$ \\
Time & 6.00 & $1,392.00$ & 39.84 & $<0.01$ \\
Group & 1.00 & 29.00 & 5.04 & 0.03 \\
Time $\times$ group & 6.00 & $1,392.00$ & 15.17 & $<0.01$ \\
\hline
\end{tabular}

numDF, degrees of freedom numerator; denDF, degrees of freedom denominator.

There were positive significant correlations between the first and the second matching for tinnitus loudness (noise-like: $r=0.77, p<0.01$; tonal: $r=0.73, p=<0.01$ ) in both groups. With respect to tinnitus/centre frequency a positive significant correlation was only observed in the tonal tinnitus group (noise-like: $r=0.14, p=0.64$; tonal: $r=0.65, p=<0.01)$.

\section{Acoustic Stimulation}

Prima facie, the stimulus IBP40 appeared to produce the strongest tinnitus suppression regardless of group and time $(M=86.16, S D=25.60)$, whereas at time point $\mathrm{T} 0$ (immediately after stimulation offset), WN40 induced the lowest tinnitus loudness $(\mathrm{M}=73.10, \mathrm{SD}=41.76)$. Descriptive statistics for the 7 utilized stimuli averaged over time and for time point $\mathrm{T} 0$ are listed in online supplementary Table S1 (see www.karger.com/doi/10.1159/000504593 for all online suppl. material) for the whole sample and divided for subgroups. Figure 2 shows the time curve for all stimuli with respect to tinnitus loudness ratings, in the same manner online supplementary Figure S1 provides information about single subject responses for each stimulus. No confounding effect caused by the order of the stimuli in the stimulation sequence was detected by our analysis $\left(t_{[1,215.60]}=0.09, p=0.93\right)$ and therefore stimuli order was not entered in the final model fitting procedure. In accordance with the previous described model fitting approach (cf. section statistical analysis in methods part), we were able to identify the following model with the best fit to our data: response $\sim$ condition + time $\times$ group $+(1 \mid i d)$. Detailed results of the model fitting are outlined in online supplementary Table S2. By testing the fixed effects of the model via expected mean square approach, significant effects for condition, time, group and for the interaction time $\times$ group on tinnitus loudness were observed (cf. Table 2). Subsequent post-hoc contrasts for condition failed to find statistically significant differences in tinnitus loudness ratings with respect to the applied stimuli (Table 3 ). 
Fig. 3. Mean suppression differences between groups. Time curve of the averaged tinnitus suppression values split for tonal and noise-like tinnitus. SD for the mean suppression data of each group is plotted as a grey ribbon. Differences between the 2 subgroups were found to be significant.

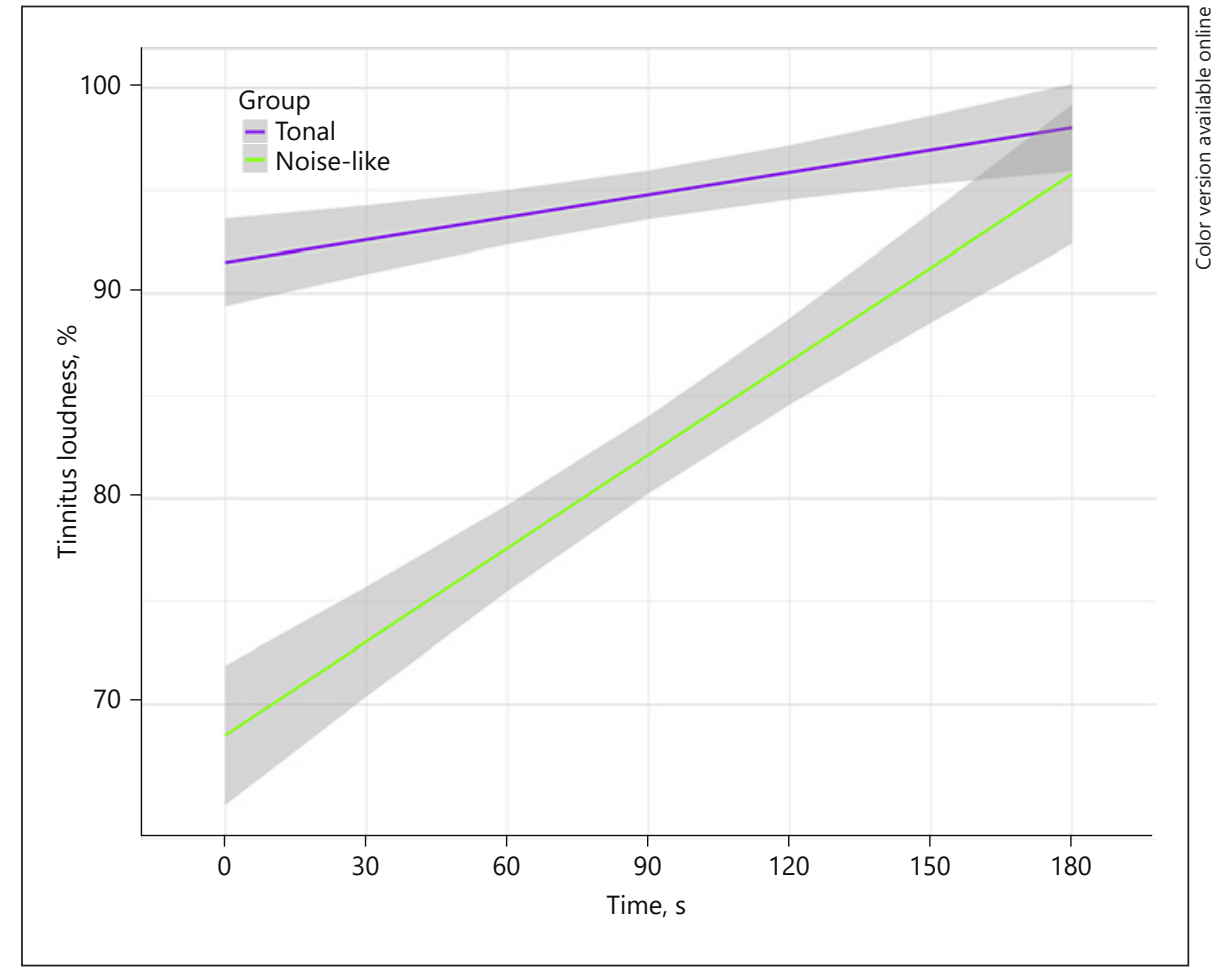

Table 3. Post-hoc Tukey contrasts for condition

\begin{tabular}{lrrr}
\hline Contrast & Estimate & \multicolumn{1}{l}{$t$} & $p$ value \\
\hline IBP - IBP10 & -1.53 & -1.06 & 0.94 \\
IBP - IBP10_MML & -4.38 & -3.05 & 0.04 \\
IBP - IBP40 & 1.08 & 0.75 & 0.99 \\
IBP - WN & -2.76 & -1.92 & 0.47 \\
IBP - WN10 & -2.17 & -1.51 & 0.74 \\
IBP - WN40 & -0.34 & -0.24 & $>0.99$ \\
IBP10 - IBP10_MML & -2.86 & -1.98 & 0.42 \\
IBP10 - IBP40 & 2.61 & 1.81 & 0.54 \\
IBP10 - WN & -1.23 & -0.86 & 0.98 \\
IBP10 - WN10 & -0.64 & -0.44 & $>0.99$ \\
IBP10 - WN40 & 1.18 & 0.82 & 0.98 \\
IBP10_MML - IBP40 & 5.47 & 3.80 & $<0.01$ \\
IBP10_MML - WN & 1.63 & 1.13 & 0.92 \\
IBP10_MML - WN10 & 2.22 & 1.54 & 0.72 \\
IBP10_MML - WN40 & 4.04 & 2.81 & 0.08 \\
IBP40 - WN & -3.84 & -2.67 & 0.11 \\
IBP40 - WN10 & -3.25 & -2.26 & 0.27 \\
IBP40 - WN40 & -1.43 & -0.99 & 0.96 \\
WN - WN10 & 0.59 & 0.41 & $>0.99$ \\
WN - WN40 & 2.41 & 1.68 & 0.63 \\
WN10 - WN40 & 1.82 & 1.27 & 0.87 \\
\hline
\end{tabular}

Degrees of freedom $=1,410.23 ; \mathrm{SE}=1.44 . \mathrm{IBP}$, individualized bandpass filtered; MML, minimum masking level; WN, white noise.
Table 4. Post-hoc Tukey contrasts for group

\begin{tabular}{llll}
\hline Contrast & Estimate & $t$ & $p$ value \\
\hline Tonal vs. noise-like & 12.65 & 2.17 & 0.04 \\
\hline \multicolumn{2}{c}{ Degrees of freedom $=31.15 ; \mathrm{SE}=5.84}$. \\
\hline
\end{tabular}

Interestingly, a significant difference in tinnitus loudness ratings between the 2 subgroups was revealed independently of condition and time as exemplified in Table 4 and Figure 3 (noise-like: $\mathrm{M}=82.14, \mathrm{SD}=26.68$; tonal: $\mathrm{M}=$ $\left.94.79, \mathrm{SD}=16.44 ; t_{[31.15]}=2.17, p=0.04\right)$. On the basis of a significant interaction among group and time, we contrasted the mean tinnitus loudness for each group for all 7 time points after stimulation. Our results point out a significant difference between the groups only at T0 (noiselike: $\mathrm{M}=63.98, \mathrm{SD}=36.49$; tonal: $\mathrm{M}=90.19, \mathrm{SD}=28.01$; $t_{[38.40]}=4.27, p<0.01 ;$ cf. Table 5).

\section{Stimulus Evaluation}

\section{Arousal}

As pointed out in online supplementary Table S3 and Figure 4, emotional stimuli evaluation for the whole group 
Fig. 4. Valence and arousal rating per stimuli. Parentheses show 95\% CI for arousal and valence ratings for all stimuli. Lowest tolerability was found in WN40 as indicated by high arousal and low valence stimulus valuation, whereas stimulus IBP10_ MML shows the highest tolerability. WN, white noise; MML, minimum masking level; IBP, individualized bandpass filtered.

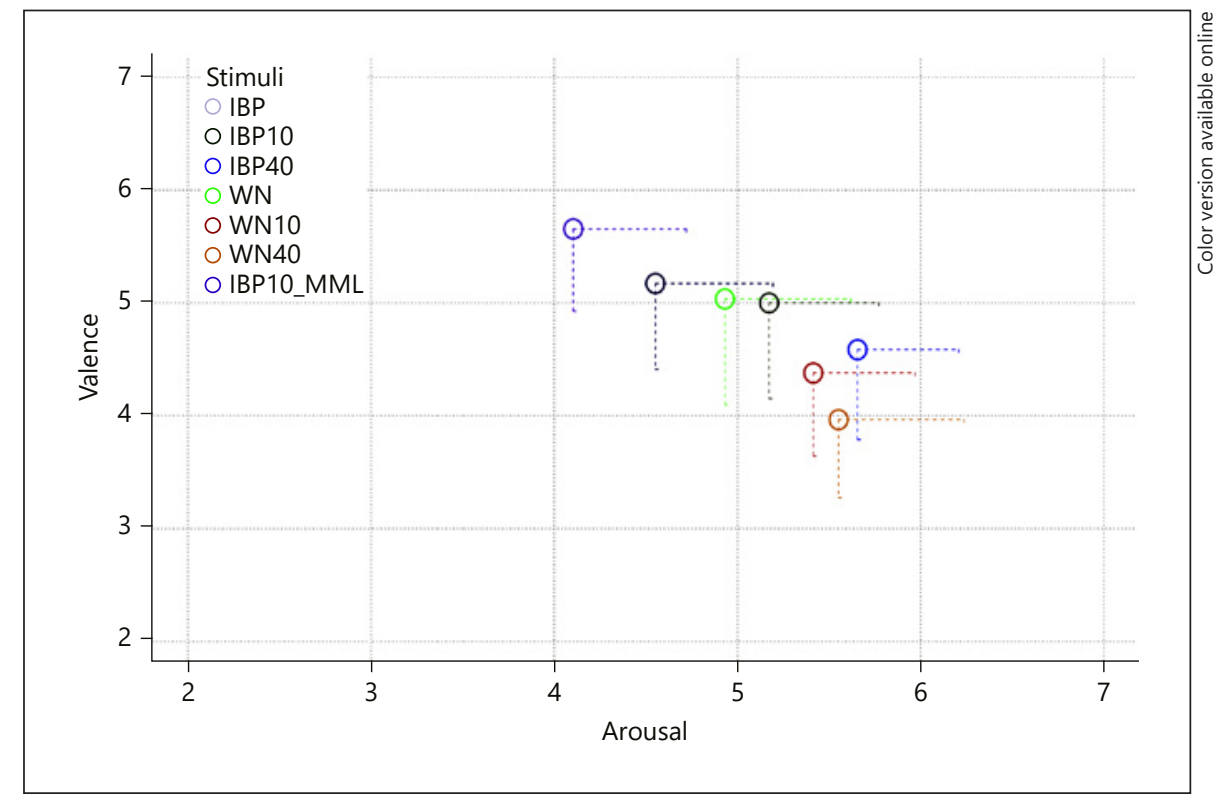

Table 5. Post-hoc Tukey contrasts for group $\times$ time

\begin{tabular}{lccc}
\hline Contrast & Estimate & $t$ & $p$ value \\
\hline $\begin{array}{l}\text { Tonal vs. noise-like } \\
\text { Time }\end{array}$ & & & \\
0 & 26.21 & 4.27 & $<0.01$ \\
30 & 20.05 & 3.27 & 0.10 \\
60 & 13.61 & 2.22 & 0.62 \\
90 & 9.91 & 1.62 & 0.93 \\
120 & 7.61 & 1.24 & $>0.99$ \\
150 & 5.54 & 0.90 & $>0.99$ \\
180 & 5.59 & 0.91 & $>0.99$ \\
\hline
\end{tabular}

Degrees of freedom $=38.40 ; \mathrm{SE}=6.13$.

identified the highest arousal ratings for stimulus IBP40, while IBP10_MML expectably manifested in the lowest arousal values. Model fitting proceedings identified the subsequent model with the best fit for our arousal data: response $\sim$ condition $+(1 \mid i d)$ (cf. online suppl. Table S4). Fixed effect testing detected a significant effect for condition (cf. Table 6). Ensuing post-hoc contrasts revealed significant differences in arousal ratings for IBP versus IBP40 $\left(t_{[180.21]}=-3.08, p=0.04\right)$, IBP10 vs. IBP10_MML $\left(t_{[180.21]}\right.$ $=2.98, p=0.05)$, IBP10_MML versus IBP40 $\left(t_{[180.21]}=\right.$ $-4.33, p<0.01)$, IBP10_MML versus WN10 $\left(t_{[180.21]}=\right.$ $-3.66, p<0.01)$, and IBP10_MML vs. WN40 $\left(t_{[180.21]}=\right.$ $-4.04, p<0.01)$. Post-hoc analysis results are reported in Table 7; relevant significant results are highlighted in bold.
Table 6. Fixed effect testing - arousal and valence

\begin{tabular}{lllll}
\hline & numDF & denDF & $F$ & $p$ value \\
\hline Arousal condition & 6.00 & 174.00 & 5.17 & $<0.01$ \\
Valence condition & 6.00 & 174.00 & 3.25 & $<0.01$
\end{tabular}

numDF, degrees of freedom numerator; denDF, degrees of freedom denominator.

\section{Valence}

In line with the descriptive arousal results, IBP10_ MML had the highest ratings for valence, whereas stimulus WN40 was evaluated with the least valence (cf. online suppl. Table S3; Fig. 4). Same model structure was fitted as for the arousal data (cf. online suppl. Table S4) and likewise a significant effect of condition was found (cf. Table 6). Post-hoc results are listed in Table 7 and demonstrate a significant difference for IBP10_MML versus $\mathrm{WN} 40\left(t_{[180.21]}=3.78, p<0.01\right)$.

\section{Discussion}

The aim of the present study was to investigate the effects of different IBP and AM noise stimuli on RI in people with tonal and noise-like tinnitus. To the best of our knowledge, no former study has systematically investigated the deployed acoustic stimulation procedures, especial- 
Table 7. Post-hoc Tukey contrasts for condition

\begin{tabular}{|c|c|c|c|c|c|c|}
\hline \multirow[t]{2}{*}{ Contrast } & \multicolumn{3}{|l|}{ Arousal } & \multicolumn{3}{|l|}{ Valence } \\
\hline & estimate & $t$ & $p$ value & estimate & $t$ & $p$ value \\
\hline IBP - IBP10 & -0.62 & -1.73 & 0.60 & 0.17 & 0.39 & $>0.99$ \\
\hline IBP - IBP10_MML & 0.45 & 1.25 & 0.87 & -0.48 & -1.08 & 0.93 \\
\hline IBP - IBP40 & -1.10 & -3.08 & 0.04 & 0.59 & 1.31 & 0.85 \\
\hline IBP - WN & -0.38 & -1.06 & 0.94 & 0.14 & 0.31 & $>0.99$ \\
\hline IBP - WN10 & -0.86 & -2.41 & 0.20 & 0.79 & 1.77 & 0.57 \\
\hline IBP - WN40 & -1.00 & -2.79 & 0.08 & 1.21 & 2.70 & 0.10 \\
\hline IBP10 - IBP10_MML & 1.07 & 2.98 & 0.05 & -0.66 & -1.47 & 0.76 \\
\hline IBP10 - IBP40 & -0.48 & -1.35 & 0.83 & 0.41 & 0.93 & 0.97 \\
\hline IBP10 - WN & 0.24 & 0.67 & 0.99 & -0.03 & -0.08 & $>0.99$ \\
\hline IBP10 - WN10 & -0.24 & -0.67 & 0.99 & 0.62 & 1.39 & 0.81 \\
\hline IBP10 - WN40 & -0.38 & -1.06 & 0.94 & 1.03 & 2.32 & 0.24 \\
\hline IBP10_MML - IBP40 & -1.55 & -4.33 & $<0.01$ & 1.07 & 2.39 & 0.21 \\
\hline IBP10_MML - WN & -0.83 & -2.31 & 0.25 & 0.62 & 1.39 & 0.81 \\
\hline IBP10_MML - WN10 & -1.31 & -3.66 & 0.01 & 1.28 & 2.86 & 0.07 \\
\hline IBP10_MML - WN40 & -1.45 & -4.04 & $<0.01$ & 1.69 & 3.78 & $<0.01$ \\
\hline IBP40 - WN & 0.72 & 2.02 & 0.41 & -0.45 & -1.00 & 0.95 \\
\hline IBP40 - WN10 & 0.24 & 0.67 & 0.99 & 0.21 & 0.46 & $>0.99$ \\
\hline IBP40 - WN40 & 0.10 & 0.29 & $>0.99$ & 0.62 & 1.39 & 0.81 \\
\hline WN - WN10 & -0.48 & -1.35 & 0.83 & 0.66 & 1.47 & 0.76 \\
\hline WN - WN40 & -0.62 & -1.73 & 0.60 & 1.07 & 2.39 & 0.21 \\
\hline WN10 - WN40 & -0.14 & -0.38 & $>0.99$ & 0.41 & 0.93 & 0.97 \\
\hline
\end{tabular}

Arousal: Degrees of freedom $=180.21 ; \mathrm{SE}=0.36$; Valence: Degrees of freedom $=180.21 ; \mathrm{SE}=0.45$. IBP, individualized bandpass filtered; WN, white noise; $\mathrm{MML}$, minimum masking level.

ly neither AM nor IBP sounds, in noise-like tinnitus cases. A parametric noise-band matching approach was applied in order to personalize BP settings in accordance with the tinnitus characteristics in the group with noise-like tinnitus, whereas the group with tonal tinnitus matched their tinnitus via the centre frequency of a fixed filter bandwidth. Taken together, all these aspects constitute novel lines of investigation within tinnitus research. Omnibus results of our experiment emphasize the ability of all used noise stimuli in inducing RI (cf. Table 2). The time courses and different suppression patterns for each stimuli appear in a similar manner as in previous studies, in that they generally converge over time after an initial maximum of suppression [Feldmann, 1983; Roberts et al., 2008; Neff et al., 2017, 2019; Vernon and Meikle, 2003; Roberts, 2007].

Contrary to our hypotheses, no statistically significant differences between the various stimuli and their impact on tinnitus perception respectively RI was observed. In more detail, neither the customization of the noise bands nor the AM resulted in significant differences between the conditions (i.e., stimuli). This outcome is in conflict with findings of earlier studies, which have suggested advantages of AM pure tones for RI [Neff et al., 2017, 2019;
Reavis et al., 2012; Tyler et al., 2014]. Yet, looking at these studies, pure tones were only compared to AM pendants with the exception of Tyler et al. [2014], who contrasted AM pure tones with unmodulated broadband noise.

A potential explanation for the lack of advantage of AM stimuli could be attributed to the circumstances, that noise is inherently composed of a wide spectrum of frequencies and signal-inherent amplitude modulation rates. These may cover up or neutralize the potential effects of certain AM rates for RI.

To the best of our knowledge, no former study specifically tested RI or sound therapies in entities with noise-like tinnitus. Of special interest, our analysis revealed statistical differences in RI for the subgroups noise-like and tonal tinnitus, with the noise-like group demonstrating larger RI than the tonal group. These significant differences were only observed immediately after the stimulation, suggesting a time-limited advantage of noise stimuli for RI in noise-like tinnitus. The reason for this group-difference is not clear, and a possible rationale may be due to physiological differences between these 2 groups with a supposed additional contribution of the extralemniscal system in noise-like tinnitus [Møller, 2006]. 
A further potential confounding factor for this group effect might be the fact that tinnitus loudness as elicited by MML, tinnitus matching and also in subjective ratings via VAS scales was found to be significant higher in the tonal subgroup. On the other hand, with no meaningful difference in HL between the groups and in consequence similar SLs, the putative confounding influence of these measures may play a negligible role. An in-depth analysis of the noise-like tinnitus group exclusively, demonstrated no statistical differences in tinnitus loudness ratings with respect to the used stimuli in a similar fashion as the analysis of the whole study sample.

However, since the bandwidth of BP filter settings in participants with tonal tinnitus was set to a range of one octave around the individual tinnitus frequency, whereas participants with noise-like tinnitus were able to individually adjust the BP filter settings, the differences in the subgroups may also derive from discrepancies in stimuli creation.

It was expected that a stimulation with noise is more pleasant or tolerable than a stimulation with pure tones. Unlike this assumption, our findings reveal a similar tolerability pattern for AM noise stimuli as Neff et al. [2019] on the basis of AM pure tones (cf. Fig. 4). The analysis conducted also show, that AM might lead to more arousal as indicated on a descriptive level as well as the significant difference between IBP and IBP40 (cf. Table 7). As must be expected, the lower intensity stimulus (IBP10 MML) had the lowest arousal and highest valence ratings.

Our results indicate that the used matching method is feasible for determining tinnitus characteristics. In detail there was good consistency for both tinnitus loudness and frequency for both matching trials in noise-like and tonal tinnitus groups. These findings are in line with Henry et al. [2013], who already reported test-retest reliability for noise-band tinnitus matching.

\section{Limitations}

The generalizability of these results is subject to certain limitations. As already discussed above, the significantly lower tinnitus loudness in the group of noise-like tinnitus could weaken our findings of subgroup differences in short-term tinnitus suppression.

However, as no difference in HL and equality in SL were observed, this may not play a significant role.

Likewise, the sample size of this experiment is rather small and gender ratio in the subgroups is unbalanced. One main issue is the impossibility to control for potential participant-related failures in noise-band matching. But for all of that, unavailable validation of the quantifica- tion of peoples' tinnitus characteristics represents a common problem in tinnitus matching approaches, as it is a subjective phenomenon. Future studies should strive for new possibilities in verifying tinnitus matching results, as well as optimization of given methodological approaches.

Since we did not compare tonal and noise stimuli, it is not possible to make a statement about a general superiority of noise stimuli in short-term tinnitus suppression in noise-like tinnitus.

\section{Conclusion}

The current study demonstrates a general efficacy of noise stimuli with different AM rates and filtering strategies for RI. Contrary to our expectations, no differences between the types of stimuli were observed. There were differences in RI among the subgroups of noise-like and tonal tinnitus, with better performance directly after the stimulation in the noise-like tinnitus group, were observed. Although, no stable rationale for the group differences can be provided, the findings may provide insights in the mechanism of RI for different tinnitus types. Future studies with larger sample sizes, improved matching/audiometry procedures and more acoustic stimulation repetitions per stimuli are needed to investigate these potential differences in more detail in order to enhance our understanding of the effects of acoustic stimulation on tinnitus perception.

Taken together these results illustrate the potential of noise-stimuli in short-term tinnitus suppression, especially in entities with noise-like tinnitus.

\section{Acknowledgement}

We want to thank Susanne Staudinger for her extremely valuable support in the collection of the data. This project was conducted as part of the European School for Interdisciplinary Tinnitus Research [Schlee et al., 2018].

\section{Statement of Ethics}

This study was approved by the Ethics Committee of the University of Regensburg, Germany (16-101-0061).

\section{Disclosure Statement}

The authors have no conflicts of interest to disclose. 


\section{Funding Sources}

S.S. received funding from the European Union's Horizon 2020 research and innovation programme under the Marie SklodowskaCurie grant (agreement number 722046). P.N. holds an Early-PostDoc Grant from the Swiss National Science Foundation (P2ZHP1 174967) and was supported by the University Research Priority Program "Dynamics of Healthy Aging" of the University of Zurich.

\section{Author Contributions}

The authors P.N., W.S., and S.S. designed the study. J.A. collected the data. S.S. and P.N. analysed the data and wrote the main manuscript. All authors contributed to and reviewed the manuscript.

\section{References}

Baguley D, McFerran D, Hall D. Tinnitus. Lancet. 2013 Nov;382(9904):1600-7.

Basile CÉ, Fournier P, Hutchins S, Hébert S. Psychoacoustic assessment to improve tinnitus diagnosis. PLoS One. 2013 Dec;8(12):e82995.

Bradley MM, Lang PJ. Measuring emotion: the self-assessment manikin and the semantic differential. J Behav Ther Exp Psychiatry. 1994 Mar;25(1):49-59.

Eggermont JJ, Roberts LE. The neuroscience of tinnitus: understanding abnormal and normal auditory perception. Front Syst Neurosci. 2012 Jul;6:53.

Eggermont JJ, Tass PA. Maladaptive neural synchrony in tinnitus: origin and restoration. Front Neurol. 2015 Feb;6:29.

Eggermont JJ. Pathophysiology of tinnitus. Prog Brain Res. 2007;166:19-35.

Erlandsson S, Dauman N. Categorization of tinnitus in view of history and medical discourse. Int J Qual Stud Health Well-being. 2013 Dec; 8(1):23530.

Farhadi M, Mahmoudian S, Saddadi F, Karimian AR, Mirzaee M, Ahmadizadeh M, et al. Functional brain abnormalities localized in 55 chronic tinnitus patients: fusion of SPECT coincidence imaging and MRI. J Cereb Blood Flow Metab. 2010 Apr;30(4):864-70.

Feldmann H. Homolateral and contralateral masking of tinnitus by noise-bands and by pure tones. Audiology. 1971 May-Jun;10(3): $138-44$.

Feldmann H. Time patterns and related parameters in masking of tinnitus. Acta Otolaryngol. 1983 May-Jun;95(5-6):594-8.

Folmer RL. Lateralization of neural activity associated with tinnitus. Neuroradiology. 2007 Aug;49(8):689-91.

Fournier P, Cuvillier AF, Gallego S, Paolino F, Paolino M, Quemar A, et al. A New Method for Assessing Masking and Residual Inhibition of Tinnitus. Trends Hear. 2018 Jan-Dec; 22:2331216518769996.

Galazyuk AV, Longenecker RJ, Voytenko SV, Kristaponyte I, Nelson GL. Residual inhibition: from the putative mechanisms to potential tinnitus treatment. Hear Res. 2019 Apr; 375:1-13.

Galazyuk AV, Voytenko SV, Longenecker RJ. Long-Lasting forward Suppression of Spontaneous Firing in Auditory Neurons: Implication to the Residual Inhibition of Tinnitus. J Assoc Res Otolaryngol. 2017 Apr;18(2):34353.
Goebel G, Berthold A, Scheffold J, Blasing L. Ein valides Screening-und Evaluationsinstrument zu Erfassung der Hyperakusisbelastung unter Berucksichtigung von Phonophobie und Rekrutiment und Schwerh origkeit. In Kongreß der Deutschen HNO-Gesellschaft. 2013; p 154.

Goebel G, Hiller W. [The tinnitus questionnaire. A standard instrument for grading the degree of tinnitus. Results of a multicenter study with the tinnitus questionnaire]. HNO. 1994 Mar; 42(3):166-72.

Hall DA, Láinez MJ, Newman CW, Sanchez TG, Egler M, Tennigkeit F, et al. Treatment options for subjective tinnitus: self reports from a sample of general practitioners and ENT physicians within Europe and the USA. BMC Health Serv Res. 2011 Nov;11(1):302.

Hallam RS, Jakes SC, Hinchcliffe R. Cognitive variables in tinnitus annoyance. Br J Clin Psychol. 1988 Sep;27(3):213-22.

Harrison XA, Donaldson L, Correa-Cano ME, Evans J, Fisher DN, Goodwin CE, et al. A brief introduction to mixed effects modelling and multi-model inference in ecology. PeerJ. 2018 May;6:e4794.

Heller AJ. Classification and epidemiology of tinnitus. Otolaryngol Clin North Am. 2003 Apr; 36(2):239-48.

Henry JA, Roberts LE, Ellingson RM, Thielman EJ. Computer-automated tinnitus assessment: noise-band matching, maskability, and residual inhibition. J Am Acad Audiol. 2013 Jun;24(6):486-504.

Kleinjung T, Fischer B, Langguth B, Sand PG., Hajak G, Dvorakova J, Eich-hammer P. Validierung einer deutschsprachigen Version des Tinnitus Handicap Inventory. Psychiat Prax. 2007;34(S 1):140-142.

Langguth B, Goodey R, Azevedo A, Bjorne A, Cacace A, Crocetti A, et al. Consensus for tinnitus patient assessment and treatment outcome measurement: Tinnitus Research Initiative meeting, Regensburg, July 2006. Prog Brain Res. 2007;166:525-36.

Langguth B, Kreuzer PM, Kleinjung T, De Ridder D. Tinnitus: causes and clinical management. Lancet Neurol. 2013 Sep;12(9):920-30.

Moazami-Goudarzi M, Michels L, Weisz N, Jeanmonod D. Temporo-insular enhancement of EEG low and high frequencies in patients with chronic tinnitus. QEEG study of chronic tinnitus patients. BMC Neurosci. 2010 Mar; 11(1):40.
Mohan A, De Ridder D, Vanneste S. Graph theoretical analysis of brain connectivity in phantom sound perception. Sci Rep. 2016 Feb;6(1): 19683.

Møller AR. Neural plasticity in tinnitus. Prog Brain Res. 2006;157:365-72.

Nakagawa S, Johnson PC, Schielzeth H. The coefficient of determination R2 and intra-class correlation coefficient from generalized linear mixed-effects models revisited and expanded. J R Soc Interface. 2017 Sep;14(134): 20170213.

Neff P, Michels J, Meyer M, Schecklmann M, Langguth B, Schlee W. $10 \mathrm{~Hz}$ Amplitude Modulated Sounds Induce Short-Term Tinnitus Suppression. Front Aging Neurosci. 2017 May;9:130.

Neff P, Zielonka L, Meyer M, Langguth B, Schecklmann M, Schlee W. Comparison of Amplitude Modulated Sounds and Pure Tones at the Tinnitus Frequency: Residual Tinnitus Suppression and Stimulus Evaluation. Trends Hear. 2019 Jan-Dec; 23: 2331216519833841.

Newman CW, Wharton JA, Shivapuja BG, Jacobson GP. Relationships among psychoacoustic judgments, speech understanding ability and self-perceived handicap in tinnitus subjects. Audiology. 1994 Jan-Feb;33(1):47-60.

Norena A, Micheyl C, Chéry-Croze S, Collet L. Psychoacoustic characterization of the tinnitus spectrum: implications for the underlying mechanisms of tinnitus. Audiol Neurotol. 2002 Nov-Dec;7(6):358-69.

Pantev C, Okamoto H, Teismann H. Music-induced cortical plasticity and lateral inhibition in the human auditory cortex as foundations for tonal tinnitus treatment. Front Syst Neurosci. 2012 Jun;6:50.

Reavis KM, Rothholtz VS, Tang Q, Carroll JA, Djalilian H, Zeng FG. Temporary suppression of tinnitus by modulated sounds. J Assoc Res Otolaryngol. 2012 Aug;13(4):561-71.

Roberts LE, Moffat G, Baumann M, Ward LM, Bosnyak DJ. Residual inhibition functions overlap tinnitus spectra and the region of auditory threshold shift. J Assoc Res Otolaryngol. 2008 Dec;9(4):417-35.

Roberts LE. Residual inhibition. Prog Brain Res. 2007;166:487-95.

Roberts LE, Moffat G, Bosnyak DJ. Residual inhibition functions in relation to tinnitus spectra and auditory threshold shift. Acta Otolaryngologica. 2006;126(sup556):27-33. 
Schaette R, König O, Hornig D, Gross M, Kempter R. Acoustic stimulation treatments against tinnitus could be most effective when tinnitus pitch is within the stimulated frequency range. Hear Res. 2010 Oct;269(1-2):95-101.

Schlee W, Hall DA, Canlon B, Cima RF, de Kleine $\mathrm{E}$, Hauck $\mathrm{F}$, et al. Innovations in Doctoral Training and Research on Tinnitus: The European School on Interdisciplinary Tinnitus Research (ESIT) Perspective. Front Aging Neurosci. 2018 Jan;9:447.
Schlee W, Hartmann T, Langguth B, Weisz N. Abnormal resting-state cortical coupling in chronic tinnitus. BMC Neurosci. 2009 Feb; 10(1):11.

Schlee W, Schecklmann M, Lehner A, Kreuzer PM, Vielsmeier V, Poeppl TB, et al. Reduced variability of auditory alpha activity in chronic tinnitus. Neural Plast. 2014;2014:436146.

Terry AM, Jones DM, Davis BR, Slater R. Parametric studies of tinnitus masking and residual inhibition. Br J Audiol. 1983 Nov;17(4): $245-56$.
Tyler R, Stocking C, Secor C, Slattery WH 3rd. Amplitude modulated S-tones can be superior to noise for tinnitus reduction. Am J Audiol. 2014 Sep;23(3):303-8.

Vernon J, Fenwick J. Identification of Tinnitus: A Plea for Standardization. J Laryngol Otol. 1984;98(S9):45-53.

Vernon JA, Meikle MB. Tinnitus: clinical measurement. Otolaryngol Clin North Am. 2003 Apr;36(2):293-305. 